\title{
Comparison of the cumulative live birth rates after one ART cycle including all subsequent frozen-thaw cycles in women undergoing IVF using progestin primed ovarian stimulation versus long GnRH agonist protocol
}

\author{
Hong Chen ${ }^{1}$, zhi qin chen ${ }^{2}$, Hung Yu Ernest $\mathrm{Ng}^{3}$, Zheng Wang ${ }^{4}$, Di Yao ${ }^{4}$, Jia Ping Pan ${ }^{2}$, \\ Junjie $\mathrm{Qu}^{4}$, Kunming $\mathrm{Li}^{1}$, and Xiao Ming Teng ${ }^{5}$ \\ ${ }^{1}$ Tongji University School of Medicine \\ ${ }^{2}$ Affiliation not available \\ ${ }^{3}$ Queen Mary Hospital / The University of Hong Kong \\ ${ }^{4}$ Tongji University Shanghai First Maternal and Infant Hospital \\ ${ }^{5}$ Shanghai First Maternity and Infant Hospital
}

July 17,2021

\begin{abstract}
Background There is scarcity of information about the cumulative live birth rates(CLBRs) and time to live birth(TTLB) between progestin primed ovarian stimulation protocol(PPOS) and long GnRH agonist protocol. Objective To compare CLBRs and TTLB in women with normal ovarian reserve following PPOS with long GnRH agonist protocol. Methods A total of 995 women who underwent IVF using either PPOS $(n=509)$ or GnRH antagonist $(n=486)$ ovarian stimulation at the discretion of the attending physicians. The primary outcome measure was the CLBRs within 18 months from the day of ovarian stimulation. Results Both groups had almost comparable demographic and cycle stimulation characteristics except for duration of infertility which was shorter in the PPOS group. CLBRs after one complete IVF cycle including fresh and subsequent FET cycles within 18 months follow up were significantly lower in the PPOS group compared that in the long agonist group 206/509 (40.5\%) and $307 / 486(63.2 \%)$, respectively (odds ratio (OR): 0.641; 95\% CI: 0.565-0.726). The average TTLB was significantly shorter in the long agonist group compared to the PPOS group $(\mathrm{P}<0.01)$. In Kaplan-Meier analysis, the cumulative incidence of ongoing pregnancy leading to LB was significantly higher in the long agonist compared in the PPOS group $(\mathrm{P}<0.001)$. Cox regression analysis revealed stimulation protocol adopted was strongly associated with the CLBRs after adjusting other confounding factors $(\mathrm{OR}=1.917$ (1.152-3.190), $\mathrm{P}=0.012)$. Conclusion PPOS offers no advantage over conventional protocol in women with a normal ovarian reserve undergoing IVF. Keywords: PPOS, long GnRH agonist protocol, IVF, CLBRs, TTLB
\end{abstract}

Comparison of thecumulative live birth rates after one ART cycle including all subsequent frozen-thaw cycles in women undergoing IVF using progestin primed ovarian stimulation versus long GnRH agonist protocol

Hong Chen ${ }^{\mathrm{a}}$, Zhi Qin Chen ${ }^{\mathrm{a}}$, Ernest Hung Yu Ng${ }^{\mathrm{b}}$, Zheng Wang ${ }^{\mathrm{a}}$, Di Yao ${ }^{\mathrm{a}}$, Jia Ping Pan ${ }^{\mathrm{a}}$, Jun Jie Qu, Kun Ming Li, Xiao Ming Teng ${ }^{\mathrm{a}}$

${ }^{a}$ Centre of Assisted Reproduction, Shanghai First Maternity and Infant Hospital, Tongji University School of Medicine, China.

${ }^{\mathrm{b}}$ Department of Obstetrics \&Gynaecology, The University of Hong Kong, Hong Kong Special Administrative Region, China. 
Correspondence address. Center of Assisted Reproduction, Shanghai First Maternity and Infant Hospital, Tongji University School of Medicine, No 536. Changle Road, 200051, Shanghai, China. Tel:(86)21-54035206; Contact: Zhi Qin Chen, Xiao Ming Teng; Email:ptchen1@hotmail.com, tengxiaoming@51mch.com.

\section{ABSTRACT}

Background

There is scarcity of information about the cumulative live birth rates(CLBRs) and time to live birth(TTLB) between progestin primed ovarian stimulation protocol(PPOS) and long GnRH agonist protocol.

Objective

To compare CLBRs and TTLB in women with normal ovarian reserve following PPOS with long GnRH agonist protocol.

Methods

A total of 995 women who underwent IVF using either PPOS $(n=509)$ or GnRH antagonist $(n=486)$ ovarian stimulation at the discretion of the attending physicians. The primary outcome measure was the CLBRs within 18 months from the day of ovarian stimulation.

Results

Both groups had almost comparable demographic and cycle stimulation characteristics except for duration of infertility which was shorter in the PPOS group. CLBRs after one complete IVF cycle including fresh and subsequent FET cycles within 18 months follow up were significantly lower in the PPOS group compared that in the long agonist group 206/509 (40.5\%) and 307/486 (63.2\%), respectively (odds ratio (OR): 0.641; 95\% CI: $0.565-0.726)$. The average TTLB was significantly shorter in the long agonist group compared to the PPOS group $(\mathrm{P}<0.01)$. In Kaplan-Meier analysis, the cumulative incidence of ongoing pregnancy leading to LB was significantly higher in the long agonist compared in the PPOS group $(\mathrm{P}<0.001)$. Cox regression analysis revealed stimulation protocol adopted was strongly associated with the CLBRs after adjusting other confounding factors $(\mathrm{OR}=1.917$ (1.152-3.190), $\mathrm{P}=0.012)$.

Conclusion

PPOS offers no advantage over conventional protocol in women with a normal ovarian reserve undergoing IVF.

Keywords: PPOS, long GnRH agonist protocol, IVF, CLBRs, TTLB

\section{INTRODUCTION}

Gonadotropin releasing hormone $(\mathrm{GnRH})$ analogues are essential in IVF to prevent a premature LH surge ${ }^{1-4}$. Inadequate suppression can cause early ovulation and affect oocyte quality and embryo development resulting in a low pregnancy rate ${ }^{5}{ }^{6}$. Despite their overall effectiveness, GnRH analogues are associated with insufficient ovarian response and cycle cancellation in $5-20 \%$ of all IVF cycles ${ }^{78}$. Furthermore, GnRH analogues have been criticized as increasing IVF protocol complexity, resulting in increased costs and the need for an HCG trigger in GnRH agonist cycles, which increases the risk of ovarian hyperstimulation syndrome ${ }^{9}$.

Concerning the adverse attributes of GnRH analogues, Kuang et al proposed the need for pituitary suppression methods that are more convenient, less costly and safer for patients. When given as cotreatment with exogenous gonadotropins for IVF, medroxyprogesterone acetate (MPA) was used in place of GnRH analogues to block the LH surge ${ }^{10}$. Prior studies indicate that compared with GnRH analogues, the use of MPA results in effective pituitary suppression with similar outcomes such as cycle cancellation rates, oocyte number and quality, fertilization rate, cleavage rate, blastocyst quality and pregnancy ${ }^{11}$. Because of the adverse effects of premature progesterone exposure on the endometrium, however, progestin cycles require a freeze-all IVF cycle with subsequent frozen embryo transfer (FET). Additionally, progestin cycles have been 
shown to require more gonadotropins compared with short GnRH agonist cycles . Several investigators have claimed that progestin cycles are more patient friendly and cost-effective ${ }^{11-17}$.

Progestins seem to provide higher pregnancy rates than the short GnRH agonist protocol following cryopreserved embryo transfers ${ }^{10} 1115$. However, in most trials, the efficacy and reproductive outcomes of PPOS regimen were compared to short GnRH agonist protocol, which is now rarely used in many assisted reproduction programs and also the live birth rate were reported by per embryo transfer rather than cumulative live birth rates (CLBRs) which can reflect the real efficacy of ovarian stimulation in ART ${ }^{18-21}$. Since many women with normal ovarian reserve are suitable for fresh embryo transfer in long agonist protocols, whether this would be the case compared with the more common long GnRH agonist protocol in which fresh transfer can be accomplished in the majority cases.

Therefore, the aim of the present study was to compare cumulative live birth rates and time to live birth in women with normal ovarian reserve following progestin primed ovarian stimulation protocol with long $\mathrm{GnRH}$ agonist protocol.

\section{Materials and methods}

\section{Patients and study design}

A retrospective study of infertile women with normal ovarian reserve attending the Assisted Reproduction clinic, Shanghai First Maternity and Infant Hospital for IVF from January 2017 to December 2019 was undertaken, and each patient was followed for 18 months from the day of the ovarian stimulation. Ethical approval was not required for the retrospective analysis.

Women were included if they fulfilled the following inclusion criteria: (i) less than 40 years of age; (ii) having indications for IVF; (iii) regular menstrual cycles over the previous 3-month period (25 - 35 days in duration); (iv) antral follicle count (AFC) of more than 5 on menstrual cycle day 2 - 3, and basal serum FSH concentration of no more than $10 \mathrm{IU} / \mathrm{L}$. Women were excluded if they had: (i) diagnosis of polycystic ovarian syndrome, (ii) an abnormal uterine cavity shown on hysterosalpingogram or hysteroscopy, (iii) moderate or severe endometriosis, (iv) use of donor eggs/sperm, (v) preimplantation genetic testing, (vi) rescue intracytoplasmic sperm injection (ICSI) or half ICSI, (vii) still having cryopreserved embryos but continuing to the next fresh IVF cycle.

Women were offered either progestin-primed ovarian stimulation protocol (PPOS group) or agonist long protocol (agonist group) at the discretion of the attending physicians or subject to the wishes of the couple.

\section{Ovarian stimulation}

Women started their IVF with ovarian stimulation using either PPOS or long agonist protocols. For the long agonist protocol, gonadotropin-releasing hormone analogue (GnRHa) (1.88mg Triptorelin acetate, Diphereline, Ipsen Pharma Biotech, France) was given for pituitary desensitisation from the mid-luteal phase in the previous cycle. On Day 2-3 of the menstrual cycle, they underwent transvaginal ultrasound examination and serum oestradiol measurement. Human menopausal gonadotrophin (hMG) (Lebaode, Lizhu, china) or recombinant FSH (Puregon, Organon, Dublin, Ireland or Gonal F, Merck Serono S.p.A, Modugno, Italy) was given at 150-225 IU per day based on the antral follicle count (AFC), age of women and previous ovarian response, according to the standard operation procedures of the centre. For the PPOS protocol, Medroxyprogesterone MPA (MPA, $10 \mathrm{mg} / \mathrm{d}$, Shanghai Xinyi Pharmaceutical Co., China) was also given from day of the ovarian stimulation until the day of ovulation trigger. Ovarian response was monitored by serial transvaginal scanning with or without hormonal monitoring. Further dosage adjustments were based on the ovarian response at the discretion of the clinicians in charge.

When three leading follicles reached [?]18 mm in diameter, Ovidrel 250 microgram (Merck Serono S.p.A., Modugno, Italy) or triptorelin (0.1 mg; Decapeptyl, Ferring Pharmaceuticals, Netherlands) and hCG (2000 IU; Lizhu Pharmaceutical Trading Co., China) were given to trigger final maturation of oocytes. Oocyte retrieval was performed around 36 hours later. 


\section{Fertilization and embryo evaluation}

Semen samples were prepared by the swim-up procedure. About 2 hours after oocyte retrieval, each oocyte was inseminated with approximately 20,000-30,000 motile spermatozoa. If the total number of motile sperm was $<10^{5}$ after washing or normal morphology was $<1 \%$, intracytoplasmic sperm injection (ICSI) was performed. Oocytes were decoronated and checked for the presence of two pronuclei to confirm fertilization. Embryos were graded on day 3 after retrieval as grade one to grade six according to the evenness of each blastomere and the percentage of fragmentation. Embryos of 6-8 cells and of grade one or two were regarded as top quality embryos. Some non-top-quality embryos were placed in extended culture until they reached the blastocyst stage.

\section{Fresh embryo transfer}

In the long agonist protocol, a maximum of two embryos was replaced on Day 3 after retrieval under transabdominal ultrasound guidance. Luteal phase support was given by vaginal or intramuscular progesterone at the discretion of the attending physicians. A pregnancy test was carried out 2 weeks after the transfer. All who had a positive pregnancy test had a transvaginal ultrasound scan 2 weeks after the positive pregnancy test (4 weeks after embryo transfer) to identify the presence of a gestation sac with a foetal heart signifying an ongoing pregnancy. All pregnant women were contacted or traced for the pregnancy outcomes after delivery or miscarriage.

\section{Cryopreservation and frozen embryo transfer (FET)}

Surplus embryos of day 3 top quality embryos or good-morphology Day 5 or 6 blastocysts in the long agonist group and all the viable embryos/blastocysts in the PPOS group were cryopreserved using vitrification. Those who did not get pregnant in the stimulated IVF cycle and those who postponed embryo transfer would undergo frozen embryo transfer (FET) at least 2 months after the stimulated cycle if they had at least one frozen embryo.

Vitrification was performed with MediCult Vitrifification Cooling (Origio, Denmark) using ethylene glycol, propylene glycol, sucrose as cryoprotectant. Embryos were vitrified one by one at room temperature. For the warming procedure following vitrification, the straw was cut and the capillary was pulled from the straw out of the liquid nitrogen, and immediately warmed one by one using MediCult Vitrification Warming (Origio, Denmark). After warming, embryos were transferred to a culture dish for evaluation and further embryo development. Only embryos with more than $50 \%$ of blastomeres present after thawing were transferred in FET cycles.

FETs were carried out in natural cycles for ovulatory women and in clomiphene induced or hormonal cycles for anovulatory women. Up to two embryos or blastocysts were transferred in FET cycles.

\section{Outcomes measures}

The primary outcome measure was the cumulative live birth rate within 18 months from the first day of ovarian stimulation. LBR which was calculated by including the first live birth generated during the one complete IVF cycle including fresh and all subsequent FET cycles.

Secondary outcome measures included incidence of premature LH surge (LH [?]10 IU/l), fertilization rate, clinical pregnancy, ongoing pregnancy, live birth rate, miscarriage, multiple pregnancy, and implantation rates in both fresh and FET cycles. Number of cycle cancellations, number of oocytes retrieved, number of obtained oocytes, number of embryos available for transfer, number of cryopreserved embryos, number of FET cycles started, moderate and severe ovarian hyperstimulation syndrome (OHSS), time to ongoing pregnancy were also compared. A baby born alive after 22 weeks gestation was classified as a live birth. Clinical pregnancy was defined as the presence of at least one gestational sac on ultrasound at 6 weeks. Ongoing pregnancy was the presence of at least one foetus with heart pulsation on ultrasound beyond 10 weeks. Miscarriage rate was defined as the number of miscarriages before 22 weeks divided by the number of women with clinical pregnancy. Fertilization rate was the percentage of zygotes with two visible pronuclei 
among inseminated oocytes. Implantation rate was calculated as the number of gestational sacs seen on scanning divided by the number of embryos replaced. Time to ongoing pregnancy leading to live birth as the time from day of ovarian stimulation to an ongoing pregnancy that led to a live birth.

We analyzed all cycles finished before 18 months after the first day of starting ovarian stimulation - whether cancelled, pregnant, or non - pregnant. To ensure validation of complete cycles, all enrolled subjects agreed to use all frozen embryos before proceeding with a new fresh IVF/ICSI cycle.

\section{Statistical analyses}

One sample of the Kolmogorov - Smirnov test was used to test the normal distribution of continuous variables. Continuous variables were given as mean $\pm \mathrm{SD}$ if normally distributed, and as median (interquartile range) if not normally distributed. Statistical comparison was carried out by Student's t-test, Mann - Whitney U-test for continuous variables and chi-square test for categorical variables, where appropriate.

Cox proportional hazard model was used to evaluate the relative prognostic significance of female age, BMI, the number of retrieved oocytes and the primary diagnosis of infertility in relation to CLBR. Regression analyses were made for the individual treatment groups in all FET cycles with transfer to evaluate the impact of independent variables on the total LBRs from FET $(\mathrm{n}=919)$.

All pregnancies within 18 months from ovarian stimulation were analyzed, whether achieved by fresh or frozen IVF cycle. The Kaplan-Meier method was used to calculate the cumulative proportion of ongoing pregnancies leading to live births, and time to pregnancy was graphically depicted by cumulative incidence curves. The log-rank test was used to measure whether significant differences existed in the cumulative incidence curves. Patients who did not reach the primary outcome (live birth) including those achieved a continuing pregnancy that did not lead to live birth were censored. Statistical analysis was performed using the Statistical Program for Social Sciences (SPSS Inc., Version 24.0, Chicago, USA). The two-tailed value of $\mathrm{P}<0.05$ was considered statistically significant.

\section{Results}

Out of 995 women who met the selection criteria, 509 women used the PPOS protocol while 486 women used the long agonist protocol. One woman in the PPOS group had premature ovulation before oocyte retrieval. No transferable embryos were available in 61 women in the PPOS group and 19 women in the long agonist group resulting in cycle cancellation, the cancellation rate was significantly higher in the PPOS group than in the agonist group $(12.0 \%$ versus $3.9 \%, \mathrm{p}<0.001)$. Within 18 mouth follow up, 76 (14.9\%)women in the PPOS group and $55(11.3 \%)$ women in the agonist group who did not achieve live birth but still have cryopreserved embryos were also included for analysis (Figure1).

\section{Demographic and the index stimulation cycle characteristics}

Baseline characteristics of two groups are presented in Table I. No significant differences were found with regard to age of women, basal AFC, basal FSH level, number of previous IVF cycles, body mass index, cause of infertility, proportion of primary infertility and insemination methods between the two groups except for duration of infertility, which was significantly shorter in the PPOS group compared to that in the long agonist group.

The starting dose of FSH was higher (225 IU versus $150 \mathrm{IU}, \mathrm{P}<0.001)$, days of stimulation is shorter $(8$ days versus 11 days, $\mathrm{P}<0.001$ ) and total FSH dose was lower (1800 IU versus $2025 \mathrm{IU}, \mathrm{P}<0.001$ ) in the PPOS group compared to those in agonist group. Serum estradiol levels $(2740 \mathrm{pg} / \mathrm{ml}$ versus $2496 \mathrm{pg} / \mathrm{ml}$, $\mathrm{P}<0.05)$ and LH level on HCG day $(2.6 \mathrm{IU} / \mathrm{ml}$ versus $0.7 \mathrm{IU} / \mathrm{ml}, \mathrm{P}<0.001)$ was higher in the PPOS groups than those in the long agonist group. However, there was no significant difference in the serum progesterone level on the hCG day between the two groups. One women in the PPOS groups experienced premature LH surge while none was seen in the long agonist group. No patient experienced OHSS in the PPOS groups, while 4 patients $(0.8 \%)$ in the long agonist group were administered into hospital due to moderate or severe OHSS. (Table 1) 
Average number of oocytes obtained (9 versus $12, \mathrm{P}<0.001$ ), number of oocytes fertilized ( 7 versus $8, \mathrm{P}<$ $0.001)$, number of cleaving embryos $(6$ versus $8, \mathrm{P}<0.001)$ and number of transferable embryos $(3$ versus $4, \mathrm{P}<0.001)$ was lower in the PPOS group as compared to that in the long agonist group. However, no differences were found in fertilization rate, cleavage rate, number of blastocyst formation and number of good quality embryos between the two groups (Table I) .

\section{Fresh embryo transfer}

In the GnRH agonist group 372 cases (77\%) completed fresh embryo transfer, resulting into 218 clinical pregnancies and 197 live birth. The clinical pregnancy rate, ongoing pregnancy, and live birth per transfer were $58.6 \%, 54.0 \%, 53.0 \%$ respectively. Seventeen $(4.6 \%)$ and four $(1.1 \%)$ women miscarried $<12$ weeks and $>12$ weeks of gestation respectively. Fresh transfer was canceled in 114 women due to elevated serum progesterone level on the trigger day, risk of OHSS, suboptimal endometrial thickness or having no transferable embryos. In the PPOS, no fresh transfer was carried out.

\section{Frozen embryo cycles}

Of all allocated patients, the total number of initiated FET cycles with thawed embryos was 665 in the PPOS group and 259 in the long agonist group. In the PPOS group, 662/665 (99.5\%) had one frozen embryo transfer compared to $257 / 259(99.2 \%)$ in the long agonist group. In the majority of FET cycles Day-3 embryos were thawed and transferred. Presence of top quality of embryos after thawing and endometrial thickness were similar between the two groups. More women had double embryo transfer in the frozen embryo cycles in the agonist group (75.5\%) than in the PPOS group (50.8\%). Hormonal cycles used for endometrium preparation were used in more FET cycles in the PPOS group 482/662 (72.8\%) compared to 104/257 (40.5\%) in the long agonist group $(\mathrm{P}<0.001)$ (Table III).

Women in the PPOS group were less likely to have a live birth following their first FET cycle 139/433 (32.1\%) compared to those in the long agonist group 85/192 (44.3\%) (OR: 1.721; 95\% CI: 0.588-0.884; P $=0.003)$. However, this difference disappeared after inclusion of additional FET cycles. Of all FET cycles, a total of 206/662 (31.1\%) cycles resulted in a live birth in the PPOS group versus $110 / 257(42.8 \%)$ in the long agonist group (OR: 0.727; 95\% CI: $0.607-0.871 ; \mathrm{P}<0.001)$. The implantation rate of total FET cycles was also lower in the PPOS group compared with that in the agonist group 293/1004 (29.2\%) and 157/455 (34.5\%) (OR: 0.846; 95\% CI: 0.721-0.992; P = 0.041) (Table IV).

In regression analyses with embryo transfer day and endometrium preparation as the independent variables, we found no impact on the total LBRs from FET for embryo transfer day (OR $=0.87 ; 95 \%$ CI 0.35-1.67; P $=0.51)$. But for endometrium preparation, we found a significant effect $(\mathrm{OR}=0.59 ; 95 \%$ CI: $0.25-0.96 ; \mathrm{P}$ $=0.03$ ) (data not shown in table). Hormonal FET cycles were correlated with lower total FET LBRs.

\section{Cumulative pregnancy and LBRs}

Cumulative pregnancy and live birth rates are listed in Table II. The CLBR after one complete IVF cycle including fresh and subsequent frozen embryo cycles within 18 months follow up were significantly lower in the PPOS group compared that in the long agonist group 206/509 (40.5\%) and 307/486 (63.2\%), respectively (odds ratio (OR): 0.641; 95\% CI: 0.565-0.726). The average time from ovarian stimulation to pregnancy and live birth was significantly shorter in the long agonist group compared to the PPOS group $(\mathrm{P}<0.001)$ (Table II). In Kaplan-Meier analysis, the cumulative incidence of ongoing pregnancy leading to live birth was significantly higher in the long agonist compared in the PPOS group. ( Log rank test, P < 0.001) (Fig. 2)

Cox proportional hazard model using the stepwise method by the women's age, stimulation protocol (PPOS/agonist), body mass index, duration of infertility, total FSH dosage, number of retrieved oocytes, causes of infertility, starting dose of FSH, days of stimulation, oestradiol and LH level on HCG day, only stimulation protocol and starting dose of FSH was entered in this model and revealed stimulation protocol adopted was strongly associated with the cumulative live birth rate after adjusting other confounding factors. $(\mathrm{OR}=1.917$ (1.152-3.190), $\mathrm{P}=0.012)$ (Table V). 


\section{Discussion}

The main finding of this study was that the CLBR in women with normal ovarian reserve after the one oocyte retrieval including fresh and all subsequent frozen embryo cycles were significant lower in the PPOS group compared with that in long agonist group, $40.5 \%$ versus $63.2 \%$ respectively. Moreover,the time to pregnancy and live birth was significantly shorter in the long agonist group compared with that in the PPOS group.

The results of the study indicated that progestins were capable of effectively preventing premature ovulation in IVF cycles. No significant difference was found in the incidence of premature LH surge and premature ovulation between the PPOS group and the long agonist group, although serum LH levels on HCG day were significantly lower in the long agonist group. The inhibitory effect of progestin on ovulation has been the basis of the design of progestin-only contraceptives, which suppress follicular growth and thus inhibit ovulation after a sustained administration. Progestin priming seems to slow the LH pulse frequency, augments the pulse amplitude and reduces the mean plasma LH concentrations compared with those in untreated women in some studies $^{22} 23$.

Progestin cycles have been shown to require more gonadotropins compared with short GnRH agonist cycles $^{11-17}$. However, in the present study we found total gonadotropin dose was lower and the day of stimulation was shorter in the PPOS group compared to that in long GnRH agonist group. This may be due to prolonged pituitary suppression in the long agonist protocol which was started from the mid-luteal phase of the previous cycle, and prolonged pituitary down-regulation by GnRHa might contribute to improved endometrial receptivity ${ }^{24}$.

In this study we found number of oocytes obtained, number of oocytes fertilized, number of cleaving embryos and number of transferable embryos was lower in the PPOS group as compared to that in the long agonist group. The results are in contrast with previous studies which showed comparable embryological characteristics in progestin and short GnRH agonist cycles $^{11-17}$. Studies with FET cycles provide an opportunity to estimate two different protocols on oocyte quality and subsequent embryo development penitential. In the first and total FET cycles, we found significantly lower clinical pregnancy and live birth rate per frozen embryo transfer as well as implantation rate in PPOS group compared to those in long agonist group. Furthermore, if we combined data from fresh and FFT cycles, the total implantation rate and pregnancy rate per transfer was still significantly lower in the PPOS group indicating the embryos originating from the PPOS protocol may have a reduced development potential to those from the long agonist group. While some researches indicate that elevated progesterone levels do not have a negative impact on the FET results of stimulated cycles using PPOS ${ }^{10} 1625$, in most trials, the efficacy and reproductive outcomes of PPOS regimen were compared to short $\mathrm{GnRH}$ agonist protocol, which is now rarely used and is recommended to be replaced by the long agonist or the antagonist protocol ${ }^{26} 27$. One randomized trial ${ }^{28}$ compared use of medroxyprogesterone versus a GnRH antagonist on the number of mature oocytes retrieved in oocyte donation cycles. Though no difference was found in the number of mature oocytes between the two groups, the clinical pregnancy rate was $31 \%$ versus $46 \%(\mathrm{P}=0.006)$ and the ongoing pregnancy rate $27 \%$ versus $40 \%(\mathrm{P}=0.015)$ for medroxyprogesterone and $\mathrm{GnRH}$ antagonists, respectively. This suggests a possible impairment of oocyte quality when medroxyprogesterone was used in ovarian stimulation.

It is difficult to directly compare our results with previous studies as none of the available study evaluated the effect of PPOS on cumulative live birth rates nor assessed time to ongoing pregnancy. In this study we report cumulative live birth rates in one complete cycle, which is the outcome of interest for infertile couples. Not only just single fresh or FET cycle live birth, but also results from one IVF cycle including all subsequent frozen embryo cycles performed within an 18-month period were evaluated,thereby giving the actual efficacy of these two strategies in the daily practice can be compared. Other strengths include none of the patients lost to follow-up in the study, leading to an increased reliability of our outcomes. Furthermore, we performed a Kaplan-Meier analysis to compare cumulative success rate in each group,as it assumed that women who did not return for subsequent FET cycles had the same chance of a pregnancy resulting in a live birth as those who returned for treatment ${ }^{19}$. Time to pregnancy was much shorter in the long agonist 
group which is also an important factor to evaluate the efficacy of IVF treatment ${ }^{29}$ and further strengthen the overall result as PPOS is not beneficial with respect to the cumulative outcomes in two groups.

Safety profile such as ectopic pregnancy rate,miscarriage rate was similar in progestin and GnRH agonist cycles. No patient experienced moderate or severe OHSS in the PPOS group owning to it is applicable for the use of a GnRHa for ovulation trigger and freezing all embryos ${ }^{30}$. In contrast, though not reaching significant difference, there were four cases of severe OHSS in the long agonist group in which HCG trigger was used and fresh embryo transfer was undertaken in the stimulated cycle. Therefore, PPOS may be more suitable for high responders but not for normal responders in whom a freeze all is likely and OHSS risk is high $^{31} 32$.

A cost-effectiveness study comparing PPOS with the short GnRH agonist and GnRH antagonist protocols suggested that PPOS was associated with significantly higher cost per live birth when conventional protocols using GnRH analogues were completed with a fresh transfer ${ }^{33}$. According to data shown in this study, we do not think that PPOS combined with an elective freeze all approach is currently justified for all IVF cycles, because avoiding a fresh transfer does not seem beneficial in the absence of a medical indication when a fresh embryo transfer is not intended ${ }^{34} 35$.

Our study is limited by its retrospective design. Although we did not calculate the sample size, around 500 cases in each group had enough power to distinguish the $20 \%$ difference of the cumulative live birth between the two groups. Cox regression analysis was carried out for controlling the basis possibly produced by imbalanced characteristics between the two groups. Further randomized trials with adequate sample size would be needed to confirm these findings.

In conclusion, in women with a normal ovarian reserve, progestin primed ovarian stimulation was associated with a lower cumulative live birth rates and a long time to pregnancy /live birth than the long agonist protocol.

\section{Acknowledgements}

We thank all the patients who participated in this study. We thank all the clinicians, embryologists, and laboratory technicians who collaborated in this study as well as the research nurses and office members for their hard work and dedication. And we are especially grateful to Prof. Ernest Hung Yu Ng for his advice and support.

\section{Authors' roles}

$\mathrm{HC}$ and ZQC were involved in the study design, execution and analysis, critical discussion and final approval of the manuscript. MXC and JPP were involved in execution and acquisition of the data. MZ contributed to execution and the analysis of the data. EHYN and KML were involved in the study design, critical discussion and revision of the manuscript. XMT was involved in supervising and coordinating the study, and final approval of the manuscript. The final manuscript and order of authorship have been approved by all authors.

\section{Funding}

This study was supported by the Special Fund for Clinical Medical Research of Shanghai Municipal Health Commission (No:202040127), General Project of Integrated Traditional Chinese and Western Medicine in Shanghai General Hospital, the National Nature Science Foundation of China (grant no. 81501319) and the Foundation of Health and Family Planning Commission of Shanghai (grant no. 201540237).

\section{Conflict of interest}

The funders had no influence on the data collection, analyses or conclusions of the study. No conflict of interests to declare. 


\section{Reference}

1. Andersen AN, Gianaroli L, Felberbaum R, et al. Assisted reproductive technology in Europe, 2001. Results generated from European registers by ESHRE. Human reproduction (Oxford, England) 2005;20(5):115876.

2. Olivennes F, Belaisch-Allart J, Emperaire J-C, et al. Prospective, randomized, controlled study of in vitro fertilization-embryo transfer with a single dose of a luteinizing hormone-releasing hormone (LH$\mathrm{RH}$ ) antagonist (cetrorelix) or a depot formula of an LH-RH agonist (triptorelin). Fertility and sterility 2000;73(2):314-20.

3. Westergaard LG, Erb K, Laursen SB, et al. Human menopausal gonadotropin versus recombinant folliclestimulating hormone in normogonadotropic women down-regulated with a gonadotropin-releasing hormone agonist who were undergoing in vitro fertilization and intracytoplasmic sperm injection: a prospective randomized study. Fertility and sterility 2001;76(3):543-9. doi: 10.1016/s0015-0282(01)01973-2 [published Online First: 2001/09/05]

4. Younis JS. "Premature luteinization" in the era of GnRH analogue protocols: time to reconsider. Journal of assisted reproduction and genetics2011;28(8):689-92. doi: 10.1007/s10815-011-9585-x [published Online First: 2011/05/27]

5. Pelinck MJ, Hoek A, Simons AH, et al. Efficacy of natural cycle IVF: a review of the literature.Human reproduction update 2002;8(2):129-39. doi: 10.1093/humupd/8.2.129 [published Online First: 2002/07/09]

6. Smacklon N, Fauser BC. Regulation of follicle development and novel approaches to ovarian stimulation for IVF. Human reproduction update 2000;6(4):307-12. doi: 10.1093/humupd/6.4.307 [published Online First: 2000/09/06]

7. van Tilborg TC, Broekmans FJ. Individualized follicle-stimulating hormone dosing and in vitro fertilization outcome in agonist downregulated cycles: a systematic review. 2016;95(12):1333-44. doi: 10.1111/aogs.13032

8. Van Tilborg TC, Torrance HL, Oudshoorn SC, et al. Individualized versus standard FSH dosing in women starting IVF/ICSI: an RCT. Part 1: The predicted poor responder.Human Reproduction 2017;32(12):2496505.

9. Toftager M, Bogstad J, Bryndorf T, et al. Risk of severe ovarian hyperstimulation syndrome in GnRH antagonist versus GnRH agonist protocol: RCT including 1050 first IVF/ICSI cycles. Human reproduction (Oxford, England)2016;31(6):1253-64. doi: 10.1093/humrep/dew051 [published Online First: 2016/04/10]

10. Kuang Y, Chen Q, Fu Y, et al. Medroxyprogesterone acetate is an effective oral alternative for preventing premature luteinizing hormone surges in women undergoing controlled ovarian hyperstimulation for in vitro fertilization.Fertility and sterility 2015;104(1):62-70.e3. doi: 10.1016/j.fertnstert.2015.03.022 [published Online First: 2015/05/10]

11. Wang Y, Chen Q, Wang N, et al. Controlled ovarian stimulation using medroxyprogesterone acetate and hMG in patients with polycystic ovary syndrome treated for IVF: a double-blind randomized crossover clinical trial. Medicine2016;95(9)

12. Zhu X, Zhang X, Fu Y. Utrogestan as an effective oral alternative for preventing premature luteinizing hormone surges in women undergoing controlled ovarian hyperstimulation for in vitro fertilization. Medicine 2015;94(21):e909. doi: 10.1097/md.0000000000000909 [published Online First: 2015/05/29]

13. Zhu X, Ye H, Fu Y. The Utrogestan and hMG protocol in patients with polycystic ovarian syndrome undergoing controlled ovarian hyperstimulation during IVF/ICSI treatments. Medicine 2016;95(28):e4193. doi: 10.1097/md.0000000000004193 [published Online First: 2016/07/20] 
14. Zhu X, Ye H, Fu Y. Use of Utrogestan during controlled ovarian hyperstimulation in normally ovulating women undergoing in vitro fertilization or intracytoplasmic sperm injection treatments in combination with a "freeze all" strategy: a randomized controlled dose-finding study of $100 \mathrm{mg}$ versus $200 \mathrm{mg}$. Fertility and sterility 2017;107(2):379-86.e4. doi: 10.1016/j.fertnstert.2016.10.030 [published Online First: 2016/11/21]

15. Zhu X, Ye H, Fu Y. Duphaston and human menopausal gonadotropin protocol in normally ovulatory women undergoing controlled ovarian hyperstimulation during in vitro fertilization/intracytoplasmic sperm injection treatments in combination with embryo cryopreservation. Fertility and sterility2017;108(3):50512.e2. doi: 10.1016/j.fertnstert.2017.06.017 [published Online First: 2017/07/13]

16. Dong J, Wang Y, Chai WR, et al. The pregnancy outcome of progestin-primed ovarian stimulation using 4 versus $10 \mathrm{mg}$ of medroxyprogesterone acetate per day in infertile women undergoing in vitro fertilisation: a randomised controlled trial.BJOG : an international journal of obstetrics and gynaecology2017;124(7):104855. doi: 10.1111/1471-0528.14622 [published Online First: 2017/03/10]

17. Yu S, Long H, Chang HY, et al. New application of dydrogesterone as a part of a progestin-primed ovarian stimulation protocol for IVF: a randomized controlled trial including 516 first IVF/ICSI cycles. Human reproduction (Oxford, England) 2018;33(2):229-37. doi: 10.1093/humrep/dex367 [published Online First: 2018/01/05]

18. Luke B, Brown MB, Wantman E, et al. Cumulative birth rates with linked assisted reproductive technology cycles. The New England journal of medicine 2012;366(26):2483-91. doi: 10.1056/NEJMoa1110238 [published Online First: 2012/06/29]

19. Maheshwari A, McLernon D, Bhattacharya S. Cumulative live birth rate: time for a consensus? Human reproduction (Oxford, England) 2015;30(12):2703-7. doi: 10.1093/humrep/dev263 [published Online First: 2015/10/16]

20. De Neubourg D, Bogaerts K, Blockeel C, et al. How do cumulative live birth rates and cumulative multiple live birth rates over complete courses of assisted reproductive technology treatment per woman compare among registries? Human reproduction (Oxford, England) 2016;31(1):93-9. doi: 10.1093/humrep/dev270 [published Online First: 2015/11/06]

21. McLernon DJ, Maheshwari A, Lee AJ, et al. Cumulative live birth rates after one or more complete cycles of IVF: a population-based study of linked cycle data from 178,898 women. Human reproduction (Oxford, England) 2016;31(3):572-81. doi: 10.1093/humrep/dev336 [published Online First: 2016/01/20]

22. SOULES MR, STEINER RA, CLIFTON DK, et al. Progesterone Modulation of Pulsatile Luteinizing Hormone Secretion in Normal Women*. The Journal of Clinical Endocrinology $\& 3$ Metabolism 1984;58(2):37883. doi: $10.1210 /$ jcem-58-2-378

23. Chabbert-Buffeta N, Skinner DC, Caraty A, et al. Neuroendocrine effects of progesterone. Steroids2000;65(10):613-20. doi:https://doi.org/10.1016/S0039-128X(00)00187-2

24. Xu B, Geerts D, Hu S, et al. The depot GnRH agonist protocol improves the live birth rate per fresh embryo transfer cycle, but not the cumulative live birth rate in normal responders: a randomized controlled trial and molecular mechanism study.Human Reproduction 2020;35(6):1306-18.

25. Lu X, Chen Q, Fu Y, et al. Elevated progesterone on the trigger day does not impair the outcome of Human Menotrophins Gonadotrophin and Medroxyprogesterone acetate treatment cycles. Scientific reports 2016;6:31112. doi: 10.1038/srep31112 [published Online First: 2016/08/09]

26. Siristatidis CS, Gibreel A, Basios G, et al. Gonadotrophin-releasing hormone agonist protocols for pituitary suppression in assisted reproduction. Cochrane Database of Systematic Reviews 2015(11)

27. Al-Inany HG, Youssef MA, Ayeleke RO, et al. Gonadotrophin-releasing hormone antagonists for assisted reproductive technology. Cochrane Database of Systematic Reviews2016(4) 
28. Begueria R, Garcia D, Vassena R, et al. Medroxyprogesterone acetate versus ganirelix in oocyte donation: a randomized controlled trial. Human Reproduction 2019;34(5):872-80.

29. Roque M, Simon C. Time to pregnancy: as important for patients as underestimated by doctors.Fertility and sterility 2020;113(3):522-23.

30. Massin N. New stimulation regimens: endogenous and exogenous progesterone use to block the LH surge during ovarian stimulation for IVF. Human reproduction update 2017;23(2):211-20. doi: 10.1093/humupd/dmw047 [published Online First: 2017/01/08]

31. La Marca A, Capuzzo M. Use of progestins to inhibit spontaneous ovulation during ovarian stimulation: the beginning of a new era? Reproductive biomedicine online2019;39(2):321-31.

32. Alexandru P, Cekic SG, Yildiz S, et al. Progestins versus GnRH analogues for pituitary suppression during ovarian stimulation for assisted reproductive technology: a systematic review and meta-analysis. Reproductive biomedicine online2020;40(6):894-903.

33. Evans MB, Parikh T, DeCherney AH, et al. Evaluation of the cost-effectiveness of ovulation suppression with progestins compared with $\mathrm{GnRH}$ analogs in assisted reproduction cycles. Reproductive biomedicine online 2019;38(5):691-98.

34. Ata B, Seli E. A universal freeze all strategy: why it is not warranted. Current Opinion in Obstetrics and Gynecology 2017;29(3):136-45.

35. Ata B, Capuzzo M, Turkgeldi E, et al. Progestins for pituitary suppression during ovarian stimulation for ART: a comprehensive and systematic review including meta-analyses. Human reproduction update 2021;27(1):48-66.

\section{Hosted file}

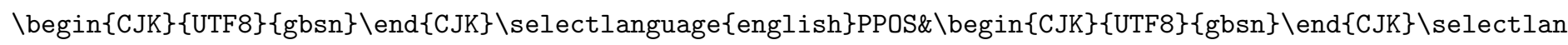
available at https://authorea.com/users/309757/articles/530771-comparison-of-the-cumulativelive-birth-rates-after-one-art-cycle-including-all-subsequent-frozen-thaw-cyclesin-women-undergoing-ivf-using-progestin-primed-ovarian-stimulation-versus-long-gnrhagonist-protocol

\section{Hosted file}

figure final.docx available at https://authorea.com/users/309757/articles/530771-comparisonof-the-cumulative-live-birth-rates-after-one-art-cycle-including-all-subsequent-frozenthaw-cycles-in-women-undergoing-ivf-using-progestin-primed-ovarian-stimulation-versuslong-gnrh-agonist-protocol 